\title{
CAPTACIÓN DE ENERGÍA LIMPIA PARA EL SUMINISTRO ELÉCTRICO
} EN LA UNTELS.

\author{
${ }^{1}$ Roberto, Pardo Caicedo Mdoi , ${ }^{1}$ Guillermo Lorenzo, Vílchez Ochoa \\ ${ }^{1}$ Universidad Nacional Tecnológica de Lima Sur, Lima, Perú
}

Recibido: 05/09/2021 Revisado: 03/10/2021 Aceptado: 16/11/2021 Publicado: 30/01/2022

\section{Resumen:}

La finalidad de este proyecto es poder brindar a la Universidad Nacional Tecnológica de Lima Sur (UNTELS) dos fuentes de energía que puedan abastecer diversas actividades. Estas fuentes serán de energía limpia y de energía convencional. La fuente de energía renovable estará compuesta por un generador fotovoltaico y un inversor; este último trabajará de manera automática para interrumpir el uso de la energía convencional cuando se esté suministrando energía limpia. Así, este trabajo de investigación pretende evaluar el dimensionamiento y la viabilidad económica para poder suministrar energía limpia a la UNTELS. Para ello, se realizó la cuantificación de la energía limpia para las actividades en la UNTELS, se estimó la viabilidad del proyecto usando indicadores económicos y se analizó la reducción de dióxido de carbono con el generador fotovoltaico. Con base en el desarrollo del proyecto, se puede afirmar que tener un sistema híbrido que aporte tanto energía convencional como energía limpia si es viable desde el punto de vista técnico y económico.

Palabras clave: Suministro de electricidad, energía solar, energía solar fotovoltaica, energía convencional, generador fotovoltaico.

\section{Resume:}

The purpose of this project is to provide the National Technological University of Lima Sur (UNTELS) with two sources of energy that can supply various activities. These sources will be clean energy and conventional energy. The renewable energy source will consist of a photovoltaic generator and an inverter; the latter will work automatically to interrupt the use of conventional energy when clean energy is being supplied. Thus, this research work aims to evaluate the dimensioning and economic viability to be able to 
supply clean energy to UNTELS. To do this, the quantification of clean energy for activities at UNTELS was carried out, the viability of the project was estimated using economic indicators and the reduction of carbon dioxide with the photovoltaic generator was analyzed. Based on the development of the project, it can be affirmed that having a hybrid system that provides both conventional energy and clean energy if it is viable from a technical and economic point of view.

Keywords: Electricity supply, solar energy, photovoltaic solar energy, conventional energy, photovoltaic generator.

\section{Introducción}

En la actualidad, a nivel mundial, consumir las energías convencionales, las cuales tienen como fuente los combustibles fósiles, está llevando al planeta a un desequilibrio ecológico, sin mencionar que estas fuentes son finitas, es decir, su disponibilidad está decreciendo y en algún momento desaparecerán. Esa es la principal razón por la cual el precio de las energías convencionales se está elevando y surge una oportunidad para dar paso a las energías renovables. El Perú está rumbo a ser un país desarrollado y el sector energético cumplirá un papel muy importante, ya que sin la electricidad no se podrán brindar productos ni servicios, así que la disponibilidad energética será un factor clave en este desarrollo. Ocurre que a mayor consumo de energía eléctrica mayor es la emisión de gases de efecto invernadero, la degradación ambiental, el desequilibrio ecológico y el consumo no responsable de recursos naturales.

El Ministerio de Energía y Minas (2018) con el objetivo de promover el uso de energía renovable y la investigación científica instaló un panel solar en la sede central, como parte de un programa piloto con propósitos fundamentales como el ahorro en el consumo de electricidad, sumarse a la iniciativa frente al cambio climático y el cuidado del ambiente. Por otro lado, El Ministerio del Ambiente (2018) inauguró el parque solar Itipampa ubicado en Moquegua con el objetivo de beneficiar a más de 350 mil hogares peruanos. Santa (2016) trabajó en el desarrollo de un sistema solar fotovoltaico para abastecer el consumo de energía en el laboratorio de Ingeniería Ambiental de la UNA LM. Debido al elevado consumo energético en el campus de la UNALM se buscó investigar la viabilidad del uso de energía solar por medio de paneles fotovoltaicos como fuente alternativa. Adicionalmente, Polo et al. (2019) analizaron los resultados obtenidos de un sistema 
fotovoltaico interconectado a la red, de $3.3 \mathrm{KW}$ de potencia, implementado en la Universidad Nacional Jorge Basadre Grohmann en la ciudad de Tacna, bajo el proyecto "Emergiendo con el sol" que tiene como objetivo monitorear la producción de energía utilizando como fuente renovable el sol.

En el ámbito internacional, Ramos y Rafael (2014) realizaron el estudio a razón del desabastecimiento energético, los cortes de energía por cableados ilegales de viviendas cercanas y los elevados gastos por los servicios energéticos, por lo que consideraron que los sistemas fotovoltaicos no abastecerán la demanda energética al $100 \%$ pero si solventarán en gran parte los gastos por la energía. Sánchez y Franco (2016) diseñaron e implementaron un sistema fotovoltaico híbrido interconectado a la red para poder abastecer el jardín botánico e incentivar el uso de energías renovables. El aporte tecnológico fue la producción de energía eléctrica de forma autónoma y a un costo bajo. Llegaron a la conclusión de que los sistemas de interconexión a la red tienen un método de instalación más fácil y práctico frente a los sistemas aislados. Además, indicaron que al utilizar inversores híbridos e inversores convencionales se puede suprimir los controladores de carga y así poder elevar la eficiencia del sistema. Fernández y Cervantes (2017) diseñaron e implementaron un sistema fotovoltaico de interconexión a la red eléctrica de la Comisión Federal de electricidad (CFE) para reducir los costos por consumo de energía eléctrica. Concluyeron que implementando el sistema fotovoltaico de interconexión eléctrica a la universidad se lograría un ahorro anual en la facturación de energía eléctrica. Alarcón y Cevallos (2019) diseñaron un sistema de iluminación eficiente a nivel fotovoltaico y comprobaron su funcionamiento. Utilizaron el luxómetro aplicando el método de punto a punto. Demostraron que luego de la instalación de los sistemas, los valores de iluminación se hallaban dentro del rango óptimo, permitiendo mejorar la calidad visual del lugar; esto en bienestar para los usuarios.

Poder dejar atrás las energías convencionales es muy complicado, pero es un cambio que es necesario, además, que hoy en día existen muchas alternativas de energías renovables. En el caso de Perú se tienen disponibles muchas de estas energías: la energía hidráulica, energía geotérmica, energía solar, energía eólica y la energía de biomasa con diferentes potenciales en todas las regiones. En este proyecto de investigación se va a trabajar con la energía solar fotovoltaica, ya que se cuenta con un gran potencial solar en toda la región; y se pretende encaminar con esta propuesta lograr una universidad sostenible que 
aporte no sólo con un ahorro significativo económico, sino también en lograr contribuir a la gran meta del siglo XXI, la sostenibilidad.

\section{Materiales y Métodos}

Entre los estudios sobre recursos energéticos renovables, Caminati et al. (2013) indican que las energías limpias, son las que tienen fuentes renovables y sostenibles, que no contaminan el ambiente con emisiones de gases de efecto invernadero (GEI) y son una opción a las frecuentemente monopolizadas. Además, las fuentes de energía renovables comprenden una emisión mínima o nula de gases contaminantes. Por otro lado, Ramírez et al. (2019) afirman que la energía solar se puede considerar como una línea relevante para un desarrollo sostenible, favoreciendo el cuidado del medio ambiente del planeta. Indican que se puede considerar el sol como una fuente inagotable, calificándola como un recurso energético renovable y esencialmente no contaminante o limpio. Dependiendo del método de transformación y aprovechamiento, se puede tener la energía solar térmica si la radiación electromagnética que proviene del sol se utiliza para el calentamiento (conversión fototérmica) y la energía solar fotovoltaica si es una conversión directa de electricidad (Caminati et al., 2013). En la Figura 1 se ilustra un esquema de la configuración general de la instalación fotovoltaica conectada a la red.

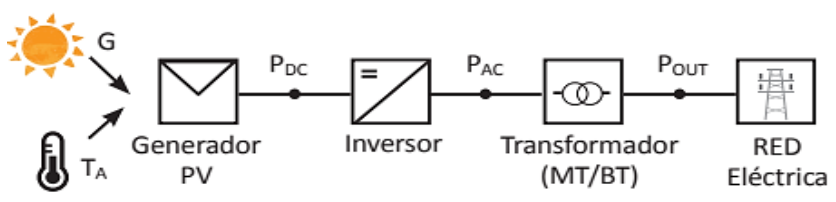

Figura 1: Configuración general de la instalación fotovoltaica conectada a la red Fuente: Alarcón, R. y Cevallos, A., 2019.

La energía solar fotovoltaica se refiere a la producción de electricidad a partir de la radiación solar, a través de los paneles fotovoltaicos. Estos paneles son de un componente semiconductor que absorbe los fotones del sol, liberando electrones, los cuales son acumulados en el panel para producir electricidad (ver Figura 2). 


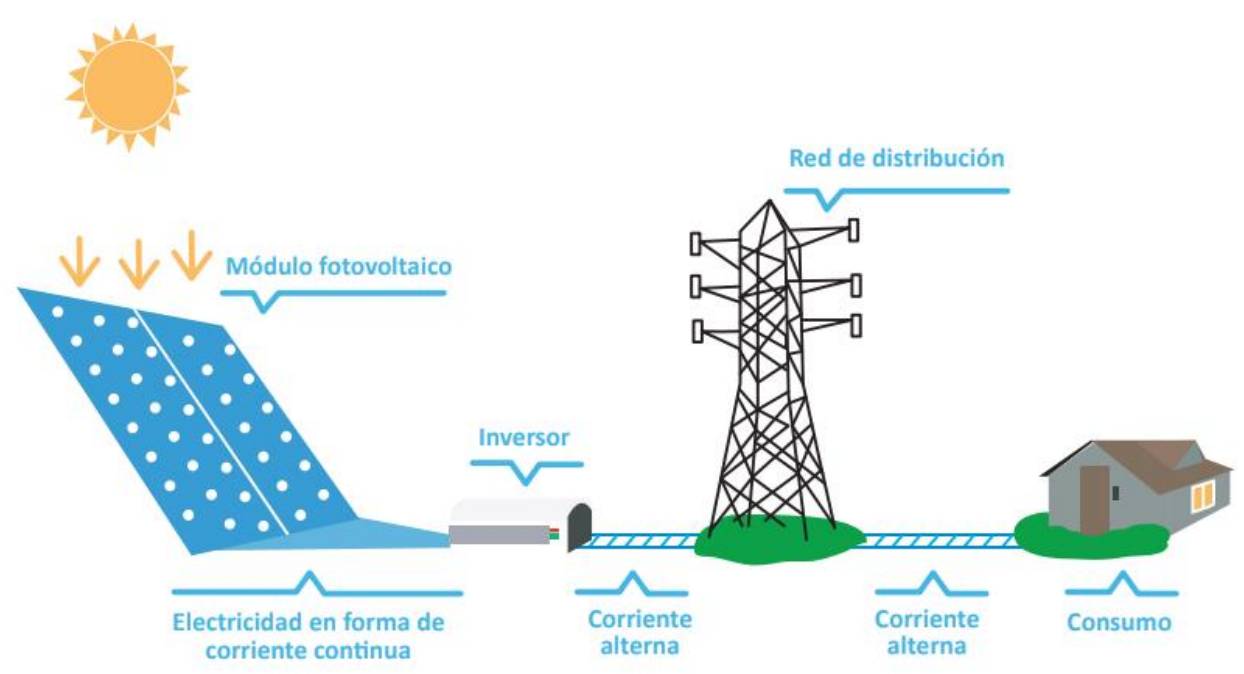

Figura 2: Formación de la energía solar fotovoltaica

Fuente: Osinergmin (2019)

Este fenómeno es conocido como efecto fotoeléctrico (Osinergmin, 2019). Según refieren Caminati et al. (2013), además de los paneles, los sistemas solares fotovoltaicos requieren baterías, reguladores de carga y un inversor de corriente. No involucran alguna actividad de combustión; tampoco generan emisiones contaminantes o ruidos molestos. Actualmente, en el Perú no hay mucha difusión de este campo por falta de normativas y políticas energéticas que incentiven el aprovechamiento de energías renovables.

Según Fernández (2018), otra fuente de energía renovable es la energía de la biomasa, que proviene de los residuos de materia orgánica de algún proceso biológico como materia orgánica de aguas residuales, lodos de depuradora, residuos sólidos orgánicos de las zonas urbanas, entre otros.

\section{Delimitación del proyecto}

En el presente trabajo de investigación se consideró la información mensual para el análisis del consumo energético desde febrero 2019 hasta enero del 2020. Se desarrolló en la azotea del pabellón administrativo en la UNTELS y se ubica en la intersección de la Av. Bolívar y la Av. Central en el distrito de Villa el Salvador, provincia de Lima y departamento de Lima. En la Figura 3 se muestra la ubicación del área de estudio. 


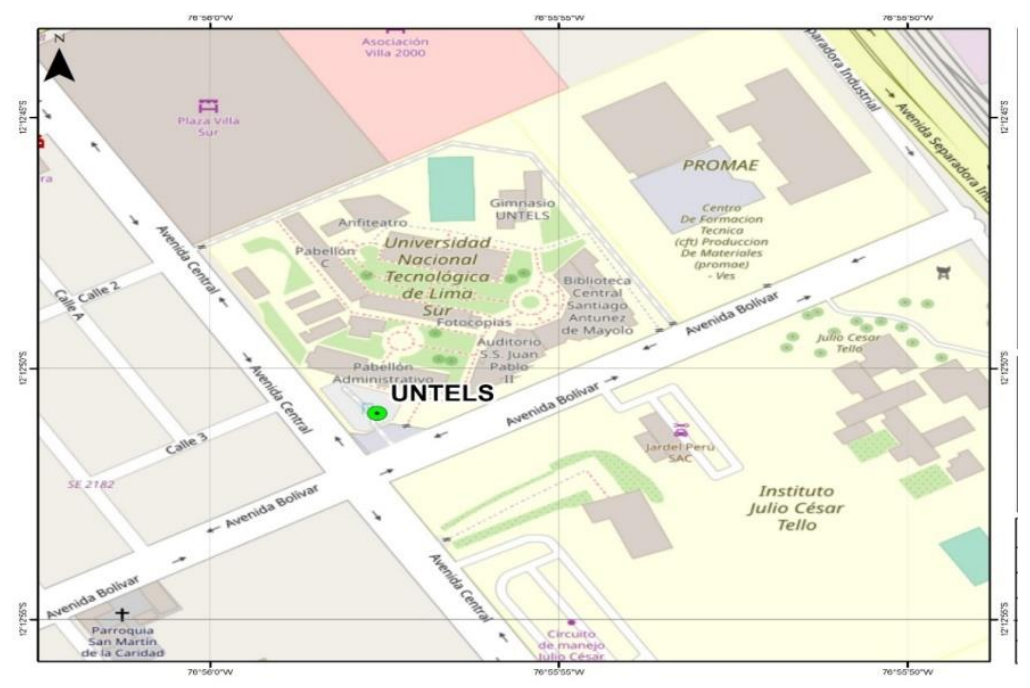

Figura 3: Localización del área de estudio

Fuente: Elaboración propia

La información disponible y necesaria para este trabajo de investigación fue la siguiente:

a. Datos de consumo energético en kilowatts hora de la UNTELS, proporcionados por la universidad, con la finalidad de conocer el gasto de energía y así poder simular un correcto dimensionamiento.

b. Datos de las horas solar pico de la UNTELS. Esto es fundamental, ya que es la cantidad de radiación que abastecerá el generador fotovoltaico y es indispensable para los cálculos del dimensionamiento. Usando las coordenadas de la universidad se descargó la información de la página Photovoltaic Geographical Information System (PVGIS).

c. Datos del área disponible. Se debe tener en cuenta este dato, ya que si el área con la que se cuenta es muy pequeña no se podría hacer un dimensionamiento muy extenso. Además, el área debe estar libre de sombras para que no exista interrupción en la captación de energía solar. Se simuló el dimensionamiento en la azotea del pabellón administrativo.

d. Datos de la sombra generada entre los paneles. Para el cálculo de sombras entre filas de paneles, fue necesario revisar la ficha técnica de los paneles para obtener sus dimensiones. 


\section{Análisis e interpretación de la información recopilada}

La información recopilada, en el presente trabajo de investigación, son datos de radiación solar y energía consumida del sistema convencional, que se han organizado en tablas para una mejor apreciación. La radiación solar dada en horas solar pico está dada por el software PVGIS (Photovoltaic Geographical Information System), donde entrega la radiación mensual a un ángulo óptimo de $15^{\circ}$ con orientación del panel hacia el norte; el promedio diario de las horas solar pico es de $4.9 \mathrm{kWh} / \mathrm{m}^{2}$. En la Tabla 1 se presenta información relacionada con la radiación solar.

Tabla 1: Radiación mensual con un ángulo de $15^{\circ}$

\begin{tabular}{llccc}
\hline N. $^{\text {o }}$ & Mes & N. $^{\text {o días }}$ & $\begin{array}{c}\text { Irradiación mensual } \\
\text { a } 15^{\circ}\end{array}$ & $\begin{array}{c}\text { Irradiación } \\
\text { diaria }\end{array}$ \\
\hline 1 & enero & 31 & 183.30 & 5.9 \\
2 & febrero & 28 & 158.80 & 5.7 \\
3 & marzo & 31 & 193.66 & 6.2 \\
4 & abril & 30 & 184.04 & 6.1 \\
5 & mayo & 31 & 147.12 & 4.7 \\
6 & junio & 30 & 107.79 & 3.6 \\
7 & julio & 31 & 117.37 & 3.8 \\
8 & agosto & 31 & 163.98 & 5.3 \\
9 & septiembre & 30 & 136.76 & 4.6 \\
10 & octubre & 31 & 142.75 & 4.6 \\
11 & noviembre & 30 & 123.96 & 4.1 \\
12 & diciembre & 31 & 134.17 & 4.3 \\
\hline
\end{tabular}

Fuente: Elaboración propia

En la Figura 4 se presenta la variación de la irradiación solar promedio mensual. 


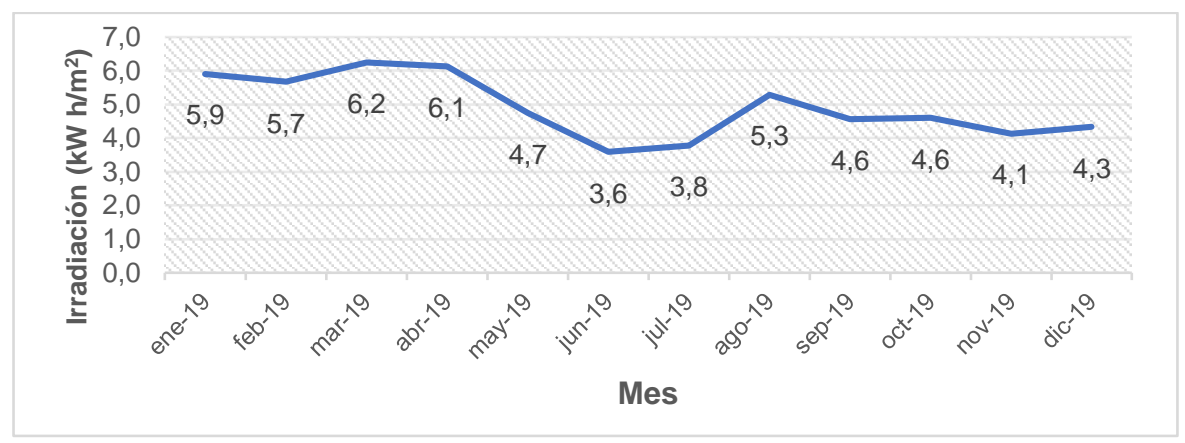

Figura 4: Gráfico de la irradiación solar mensual

Fuente: PVGIS, 2020

Se organizó, como se muestra en la Tabla 2, el consumo mensual de la universidad en kWh, ya que éste se consideró como el punto de partida para el correcto cálculo de los paneles solares.

Tabla 2: Consumo mensual del sistema convencional en la UNTELS en kWh

\begin{tabular}{lccc}
\hline N. $^{\circ}$ & Año & Mes & Consumo kWh-mes \\
\hline 1 & 2019 & febrero & 26928 \\
2 & 2019 & marzo & 27576 \\
3 & 2019 & abril & 35268 \\
4 & 2019 & mayo & 37212 \\
5 & 2019 & junio & 39942 \\
6 & 2019 & julio & 34524 \\
7 & 2019 & agosto & 22824 \\
8 & 2019 & septiembre & 37254 \\
9 & 2019 & octubre & 37788 \\
10 & 2019 & noviembre & 35196 \\
11 & 2019 & diciembre & 29418 \\
12 & 2020 & enero & 17508 \\
\hline Consumo promedio mensual kWh-mes & 31787 \\
Consumo promedio diario kWh-día & 1045 \\
\hline
\end{tabular}

Fuente: Elaboración propia

\section{Determinación y análisis del problema}

La Universidad Nacional Tecnológica de Lima Sur (UNTELS) cuenta actualmente con un sistema de abastecimiento de energía eléctrica convencional proporcionado por la 
empresa Luz del Sur, el cual obtiene su fuente de energía eléctrica principalmente de centrales hidroeléctricas y de centrales térmicas que provocan un desequilibrio ecológico y aportan al ambiente una gran cantidad de gases de efecto invernadero, respectivamente. En este trabajo lo que se busca es producir la energía eléctrica, mediante un sistema fotovoltaico, proporcionada de fuente de energía solar, que permitirá tener un sistema híbrido, que funcione tanto con energía convencional como renovable. Con la energía renovable se busca abastecer un $2 \%$ del consumo total de la universidad. Esto conlleva a que las actividades realizadas en la UNTELS tengan como fin optimizar la energía convencional y hacer uso de la energía solar. Así, se tendrá un sistema con energía convencional y energía renovable para el abastecimiento de energía eléctrica en la UNTELS.

\section{Modelo de solución propuesto}

Según Tamayo (como se citó en Osinergmin, 2019), gran parte del territorio peruano cuenta con un óptimo recurso de energía solar por su cantidad y uniformidad durante el año. Por esta razón, en este trabajo se propone para la UNTELS un generador fotovoltaico para la captación y suministro de energía limpia, el cual se dimensionará con componentes reconocidos en el mercado solar por su calidad, fiabilidad técnica y económica.

\section{Elección de componentes}

Panel solar: Los módulos fotovoltaicos serán de la marca Jinko Solar del modelo JKM335PP-72, tipo policristalino, con una potencia de $335 \mathrm{~W}$. Sus células fotovoltaicas de alta eficiencia y vidrio texturizado de alta transmisión permiten que el módulo minimice los costos y maximice su producción energética. La calidad del marco de aluminio del módulo es resistente a la corrosión y superligero, asegurando estabilidad en los módulos. 
Inversor: Se contará con un inversor de la marca Fronius del modelo Fronius Symo 6.0-3-M, con una potencia de $6 \mathrm{~kW}$. Éste se encargará de transformar la corriente continua del generador fotovoltaico a corriente alterna para que pueda ser suministrada a la red de baja potencia para, así, disminuir el gasto de la facturación mensual en la UNTELS.

\section{Dimensionamiento}

Para dimensionar de forma correcta se debe definir la carga promedio de energía diaria que se desea abastecer. En este caso, la universidad tiene un consumo promedio de $1045.04 \mathrm{kWh}$ y la finalidad es abastecer con energía limpia el $2 \%$ del consumo diario, lo cual equivale a 20.9 kWh. Esta información se tomó de los últimos doce recibos de luz de la universidad, con actividades normales, ya que por el estado de emergencia sanitaria que comenzó en el país, las actividades empezaron a disminuir desde febrero del año 2020, razón por lo que se tomó la información desde febrero del 2019 hasta enero del 2020.

Adicionalmente, fue necesario determinar hacia dónde estarán orientados los paneles y el ángulo de inclinación que éstos tendrán respecto a la superficie horizontal. Esto con la finalidad de que los paneles puedan captar la mayor cantidad de energía solar. El ángulo de inclinación del módulo será $15^{\circ}$, ya que la universidad se encuentra en la latitud $12^{\circ}$ $12^{\prime} 50.20$ ” y la orientación debe ser apuntando hacia la línea ecuatorial; como el área de estudio se encuentra en el hemisferio sur, la cara del panel deberá estar orientada hacia el norte.

En la Tabla 3 se presenta información sobre el ángulo de inclinación del módulo en función de la latitud.

Tabla 3: Ángulo de inclinación vs latitud

\begin{tabular}{ll}
\hline Latitud del lugar & \multicolumn{1}{c}{$\begin{array}{c}\text { Ángulo de } \\
\text { inclinación del } \\
\text { módulo }\end{array}$} \\
\hline De $0^{\circ}$ a $15^{\circ}$ & $15^{\circ}$ \\
De $15^{\circ}$ a $25^{\circ}$ & El mismo que la \\
& latitud
\end{tabular}




\begin{tabular}{ll}
\hline De $25^{\circ}$ a $30^{\circ}$ & Latitud $+5^{\circ}$ \\
De $30^{\circ}$ a $35^{\circ}$ & Latitud $+10^{\circ}$ \\
De $35^{\circ}$ a $40^{\circ}$ & Latitud $+15^{\circ}$ \\
Más de $40^{\circ}$ & Latitud $+20^{\circ}$ \\
\hline
\end{tabular}

Fuente: Caicedo y Torres (2020)

Teniendo la información del consumo mensual en kWh de la UNTELS (ver Tabla 2) el diseño pretende cubrir $20.9 \mathrm{kWh}$ que corresponde a un $2 \%$ del promedio diario del consumo. Con base en esta información, se procedió a calcular el número de paneles solares necesarios para cubrir esta demanda, empleando la siguiente ecuación

$\mathrm{N}_{\text {paneles }}=\frac{\mathrm{E}_{\text {diaria }} * 125 \%}{\mathrm{P}_{\text {panel }} * \mathrm{H}_{\mathrm{sp}} * \mathrm{n}_{\text {inversor }}}$

en la cual $\mathrm{N}_{\text {paneles }}$ corresponde al número de paneles, $\mathrm{E}_{\text {diaria }}$ es la energía promedio diaria consumida por la UNTELS, en este caso se cubrirá el $2 \%, \mathrm{P}_{\text {panel }}$ es el potencial del panel fotovoltaico, $\mathrm{H}_{\mathrm{sp}}$ representa las horas solar pico (relacionada con la cantidad de energía solar que incide en una hora por metro cuadrado de superficie) y $\mathrm{n}_{\text {inversor }}$ es la eficiencia del inversor.

Como medida de protección el $125 \%$ significa el sobredimensionamiento para asegurar la cantidad de paneles y en el caso del inversor, la eficiencia tiene un rango que va desde 0.8 hasta 0.9. En este caso se tomó el valor de 0.9. La información de las horas solar pico se puede corroborar en la página de Photovoltaic Geographical Information System (PVGIS). Este dato es importante para estimar la potencia generada por los paneles fotovoltaicos (Valdiviezo, 2014). Se puede apreciar que la irradiación solar acumulada diaria es de $4.9 \mathrm{kWh} / \mathrm{m}^{2}$ (ver Tabla 1 ). Así, se tendrán 


$$
\mathrm{N}_{\text {paneles }}=\frac{20.9 * 125 \%}{335 * 4.9 * 0.9}=17.7
$$

Después de realizado el cálculo, se obtuvo que el número de paneles requeridos es 17.7. Así, se dimensionó el abastecimiento con 18 paneles solares de la marca Jinko Solar, cada uno con una potencia de $335 \mathrm{~W}$, lo cual entregará una potencia total de $6030 \mathrm{~W}$. Una vez dimensionada la cantidad de paneles requeridos, se calculó la potencia que entrará al inversor. Para poder elegirlo, se utilizó la siguiente ecuación

$$
\text { Potencia de entrada }=\mathrm{V}_{\mathrm{mp}} * \mathrm{I}_{\mathrm{mp}} * \mathrm{~N}_{\text {paneles }}
$$

donde Potencia de entrada es la potencia que entrará al inversor, $\mathrm{V}_{\mathrm{mp}}$ es la máxima capacidad de voltaje del panel, $\mathrm{I}_{\mathrm{mp}}$ es la máxima capacidad de corriente del panel y $\mathrm{N}_{\text {paneles }}$ es el número de paneles a instalar.

Así, aplicando la ec. 2 se obtiene que

$$
\text { Potencia de entrada }=38 * 8.82 * 18=6032.88 \mathrm{~W}
$$

La potencia de entrada resultante al inversor es $6032.88 \mathrm{~W}$. El inversor seleccionado tiene una capacidad de $6000 \mathrm{~W}$; no existirá problema ya que el inversor tiene la capacidad de aguantar un $20 \%$ de sobrecarga.

Posteriormente, se calculó el número de paneles en serie y en paralelo. Para hallar el número de paneles en serie la suma de sus voltajes no debe exceder el voltaje de entrada del inversor, y para hallar el número de paneles en paralelo la suma de su amperaje no debe exceder el amperaje de entrada del inversor.

La tensión resultante al conectar los paneles en serie debe ser menor que la tensión de entrada del inversor. Así, considerando 9 paneles en serie se obtendrá

Tensión de entrada del inversor $>V_{m p}$ Paneles en serie 


$$
\begin{gathered}
800>38 * 9 \\
800>342
\end{gathered}
$$

$\mathrm{Al}$ cumplir con este requisito, se da conformidad para conectar 9 paneles en serie.

La corriente debe ser la suma de las corrientes del panel multiplicado por las filas de paneles en paralelo y ésta no debe exceder a la corriente de entrada del inversor. Así, considerando 2 filas de paneles en paralelo

Corriente de entrada del inversor $>I_{m p}$ Paneles en paralelo

$$
\begin{gathered}
18>8.82 * 2 \\
18>17.64
\end{gathered}
$$

Como se cumple con este requisito, se da conformidad para conectar 2 filas de paneles en paralelo. La instalación eléctrica del sistema fotovoltaico comenzará en el generador fotovoltaico que estará compuesto de 18 paneles de $335 \mathrm{~W}$, con 2 filas en paralelo y cada fila tendrá 9 paneles en serie, los cuales emitirán corriente continua. Después esta corriente pasará por el inversor de $6 \mathrm{~kW}$ para ser transformada en corriente alterna; después esta corriente pasará por un tablero de protección para, posteriormente, abastecer en promedio el $2 \%$ del consumo total en la universidad. Para proteger la integridad del generador fotovoltaico, al final de cada ramal, se colocarán fusibles y diodos de bloqueo a modo de protección.

Luego, se calculó, según la ec. 5, la producción mensual de energía renovable que brindará el generador fotovoltaico, ya que la radiación es un factor determinante en este trabajo de investigación.

Producción $\mathrm{kWh}-$ mes $=\mathrm{P}_{\mathrm{GF}} * \mathrm{H}_{\mathrm{sp}} * \mathrm{~N}_{\text {mes }} * \mathrm{n}_{\text {inversor }}$

en la cual Producción kWh - mes es la producción mensual de energía renovable, $\mathrm{P}_{\mathrm{GF}}$ es la potencia del generador fotovoltaico en Watts, $\mathrm{H}_{\mathrm{sp}}$ representa las horas solar pico, $\mathrm{N}_{\text {mes }}$ es el número de días del mes y $\mathrm{n}_{\text {inversor }}$ es la eficiencia del inversor. En la Tabla 4 se muestra la producción mensual de energía renovable. 
Tabla 4: Producción de kWh-mes de energía renovable

\begin{tabular}{|c|c|c|c|c|c|c|}
\hline $\mathrm{N}^{\circ}$ & Mes & $\mathbf{N}_{\text {mes }}$ & $\mathbf{H}_{\mathbf{s p}}$ & $\mathbf{n}_{\text {inversor }}$ & $\mathbf{P}_{\mathrm{GF}}$ & $\begin{array}{l}\text { Producción de } \\
\text { energía renovable } \\
\text { kWh-mes }\end{array}$ \\
\hline 1 & enero & 31 & 5.9 & 0.9 & 6030 & 994.77 \\
\hline 2 & febrero & 28 & 5.7 & 0.9 & 6030 & 861.81 \\
\hline 3 & marzo & 31 & 6.2 & 0.9 & 6030 & 1050.99 \\
\hline 4 & abril & 30 & 6.1 & 0.9 & 6030 & 998.79 \\
\hline 5 & mayo & 31 & 4.7 & 0.9 & 6030 & 798.42 \\
\hline 6 & junio & 30 & 3.6 & 0.9 & 6030 & 584.98 \\
\hline 7 & julio & 31 & 3.8 & 0.9 & 6030 & 636.97 \\
\hline 8 & agosto & 31 & 5.3 & 0.9 & 6030 & 889.92 \\
\hline 9 & $\begin{array}{c}\text { septiembr } \\
\mathrm{e}\end{array}$ & 30 & 4.6 & 0.9 & 6030 & 742.20 \\
\hline 10 & octubre & 31 & 4.6 & 0.9 & 6030 & 774.70 \\
\hline 11 & $\begin{array}{c}\text { noviembr } \\
\mathrm{e}\end{array}$ & 30 & 4.1 & 0.9 & 6030 & 672.73 \\
\hline 12 & diciembre & 31 & 4.3 & 0.9 & 6030 & 728.14 \\
\hline
\end{tabular}

Fuente: Elaboración propia

De la información de la Tabla 4, se observa que se tendrá una producción promedio mensual de $811.20 \mathrm{kWh}$ y una producción anual de $9734.41 \mathrm{kWh}$. En la Figura 5 se muestra cómo varía la producción de energía renovable, ya que la radiación recibida no es constante, pero en ningún momento se dejará de producir menos del $2 \%$ de la energía propuesta.

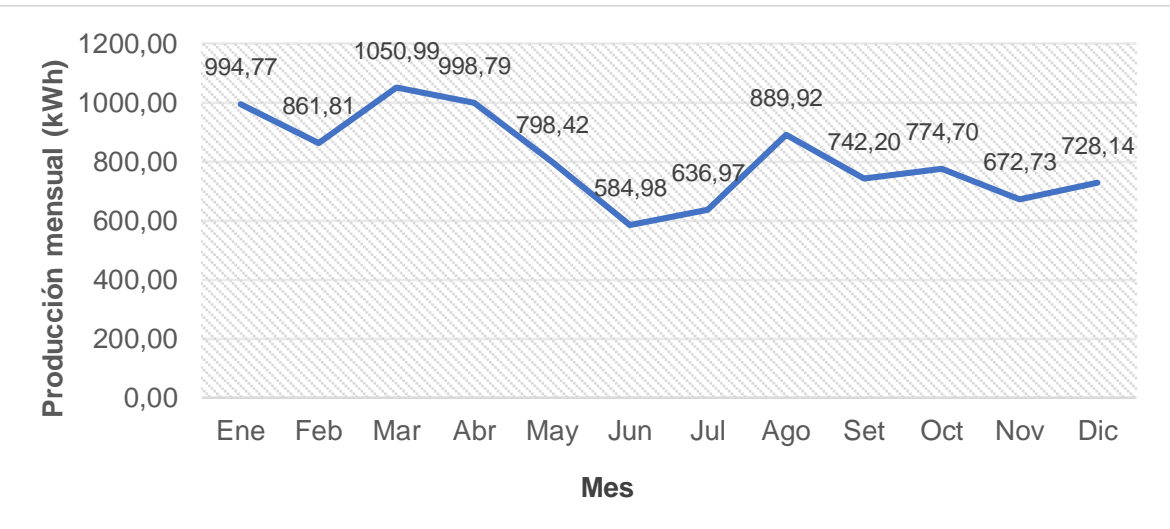


Figura 5: Gráfico de la producción mensual de energía renovable

\section{Fuente: Elaboración propia}

En el desarrollo de este tipo de proyecto, hay que tener en cuenta todo lo relacionado con los gases de efecto invernadero (GEI), los cuales son componentes gaseosos que se localizan en la atmósfera y conservan parte de la energía que el suelo libera. Este proceso es conocido como "efecto invernadero". Es un fenómeno natural que estabiliza la temperatura entre frío y caliente de la tierra haciendo posible el desarrollo de vida en ella. Según Arias (2018), el dióxido de carbono $\left(\mathrm{CO}_{2}\right)$ es un gas de efecto invernadero producido por el uso de combustible fósil (petróleo, gas, carbón, etc.) y por el cambio de uso de la tierra (deforestación). Este gas ha contribuido a mantener una temperatura constante dentro de la tierra, sin embargo, en la actualidad es responsable de casi el $76 \%$ del calentamiento global previsto para los próximos años.

Según Pandey, Agrawal y Pandey, 2010 menciona que la huella de carbono es un indicador que expresa los gases de efecto invernadero (GEI) emitidos a la atmósfera como consecuencia de las actividades para la generación o consumo de bienes o servicios en todos los procesos de su ciclo de vida y se expresa en kilogramos de dióxido de carbono $\left(\mathrm{CO}_{2}\right)$. Se definen 3 alcances por cuestiones de reporte y contabilidad de GEI: Alcance 1 que corresponde a emisiones directas de GEI. Se entiende como las emisiones de fuentes que pertenecen a la empresa. Luego, el Alcance 2 se refiere a las emisiones indirectas de GEI asociadas a la electricidad. Contiene las emisiones debido a la producción de energía adquirida y de consumo para la organización y el Alcance 3 que corresponde a otras emisiones indirectas. Para empezar el cálculo de la huella de carbono, se deben expresar los gases de efecto invernadero en una unidad equivalente en $\mathrm{Kg}$ de $\mathrm{CO}_{2}$ :

- $\mathrm{CH}_{4}=25 \mathrm{Kg}$ de $\mathrm{CO}_{2}$

- $1 \mathrm{~kg} \mathrm{~N}_{2} \mathrm{O}=298 \mathrm{Kg} \mathrm{CO}_{2}$

Cuando todos los valores de GEI estén en una misma unidad se pueden resumir y expresar como $\mathrm{CO}_{2}$ equivalente ( $\left.\mathrm{CO} 2 \mathrm{eq}\right)$. El factor de emisión permite contabilizar las emisiones de GEI a partir de reseñas de actividades disponibles (por ejemplo: kWh de energía consumida) y las emisiones totales de GEI. Depende del tipo de elemento; para esta 
investigación depende del tipo de combustible a trabajar. En la Tabla 5 se presentan los valores del factor de emisión en función del tipo de combustible.

Tabla 5: Factor de emisión

\begin{tabular}{ccc}
\hline Tipo de combustible & $\begin{array}{c}\text { Factor de emisión } \\
(\boldsymbol{k g C O 2} \boldsymbol{e q} / \boldsymbol{k W h})\end{array}$ & $\begin{array}{c}\text { Factor de emisión } \\
(\boldsymbol{t C O 2} \boldsymbol{e q} / \boldsymbol{k W h})\end{array}$ \\
\hline Diesel & 9.7 & 0.002560 \\
Gasolina & 7.9 & 0.002090 \\
Electricidad & 0.615 & 0.000615 \\
\hline
\end{tabular}

Fuente: Ministerio de Energía y Minas, 2017

La fórmula a emplear para el cálculo de $\mathrm{CO}_{2}$ eq, se resume en:

$\mathrm{CO}_{2}$ eq $=$ Datos de cada elemento $\mathrm{x}$ Factor de emisión

Por lo tanto, se puede determinar la reducción en la huella de carbono mediante la siguiente ecuación

kgCO2eq - mes = Producción del kWh - mes x Factor de emisión (kgCO2eq/ $\mathrm{kWh})(7)$

Guzmán et al. (2016) mencionan que, actualmente, se incentiva la búsqueda de nuevas opciones de energías renovables para menguar el cambio climático y conseguir sistemas de generación de energías sostenibles; se tiene la energía solar, de biomasa y otras fuentes renovables que permiten el secuestro del carbono y la adaptabilidad al cambio climático. Los proyectos de generación eléctrica que tienen como fuente recursos renovables han mitigado un aproximado de 6.4 millones de toneladas de $\mathrm{CO}_{2}$ eq en un periodo de 8 años contando desde el 2008, obteniendo una mitigación del $37 \%$ de emisiones de $\mathrm{CO}_{2}$.

Así, considerando lo anteriormente mencionado, una vez obtenida la producción mensual de energía renovable en $\mathrm{kWh}$, se procedió a calcular la cantidad de dióxido de carbono equivalente para poder hallar el ahorro en la huella de carbono, ya que se empezará a suministrar un porcentaje de energía limpia mensual para el consumo en la universidad. Usando el factor de emisión que brinda el alcance 2, del protocolo de gases de efecto 
invernadero, se logra calcular la reducción de dióxido de carbono equivalente. Este factor se usará cuando la fuente sea generación eléctrica adquirida o consumida. De la Tabla 5 se obtiene la información sobre el factor de emisión de dióxido de carbono equivalente, del alcance 2 del protocolo de GEI, para el caso de electricidad como tipo de combustible.

En la Tabla 6 se presenta el cálculo mensual del dióxido de carbono equivalente que se podrá dejar de emitir en la universidad.

Tabla 6: Cálculo de dióxido de carbono equivalente

\begin{tabular}{ccccc}
\hline N. & Mes & $\begin{array}{c}\text { Producción del } \\
\text { generador kWh- } \\
\text { mes }\end{array}$ & $\begin{array}{c}\text { Factor de emisión } \\
(\boldsymbol{k g C O} \text { eq } / \boldsymbol{k W h})\end{array}$ & $\begin{array}{c}\boldsymbol{k g C O 2} \boldsymbol{e q} \text { - } \\
\text { mes }\end{array}$ \\
\hline 1 & enero & 994.77 & 0.615 & 611.78 \\
2 & febrero & 861.81 & 0.615 & 530.01 \\
3 & marzo & 1050.99 & 0.615 & 646.36 \\
4 & abril & 998.79 & 0.615 & 614.25 \\
5 & mayo & 798.42 & 0.615 & 491.03 \\
6 & junio & 584.98 & 0.615 & 359.76 \\
7 & julio & 636.97 & 0.615 & 391.73 \\
8 & agosto & 889.92 & 0.615 & 547.30 \\
9 & septiembre & 742.20 & 0.615 & 456.45 \\
10 & octubre & 774.70 & 0.615 & 476.44 \\
11 & noviembre & 672.73 & 0.615 & 413.73 \\
12 & diciembre & 728.14 & 0.615 & 447.81 \\
\hline
\end{tabular}

Fuente: Elaboración propia

En la Figura 6 se presenta información sobre la reducción del dióxido de carbono equivalente mensual, que se tendrá en la universidad, como resultado del consumo de energía solar. 


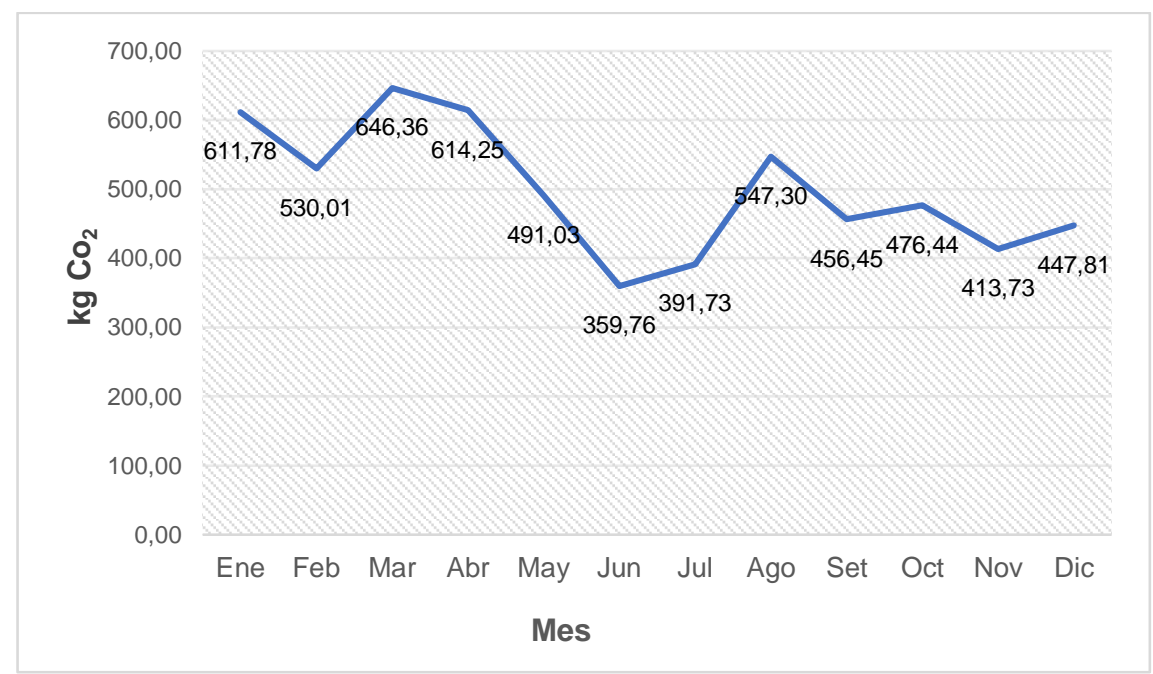

Figura 6: Gráfico del dióxido de carbono equivalente mensual que se podrá reducir en la universidad

Fuente: Elaboración propia

Se puede interpretar que se tendrá una reducción promedio mensual de $498.89 \mathrm{~kg} \mathrm{CO}_{2}$

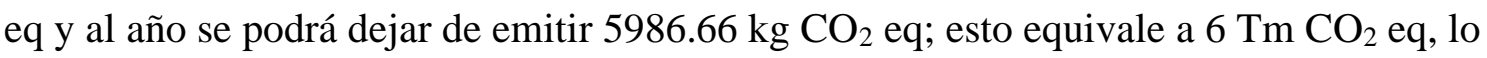
cual es una cantidad considerable, ya que el sistema solo abastecerá en promedio un $2 \%$ del consumo total.

\section{Análisis económico}

Antes de empezar con el análisis de los indicadores económicos, se debe tener en cuenta el precio por kWh que cobran por el sistema convencional y la tasa de crecimiento anual en el cobro por kWh por parte de las empresas de energía eléctrica convencional. Según indica Plasencia en 2018, el costo de energía eléctrica por kWh del sistema convencional es de 0.5939 soles. López, 2019 indica que la tasa de crecimiento anual en el costo de energía eléctrica del sistema convencional es 0.0339 soles, como se observa en la Figura 7. 


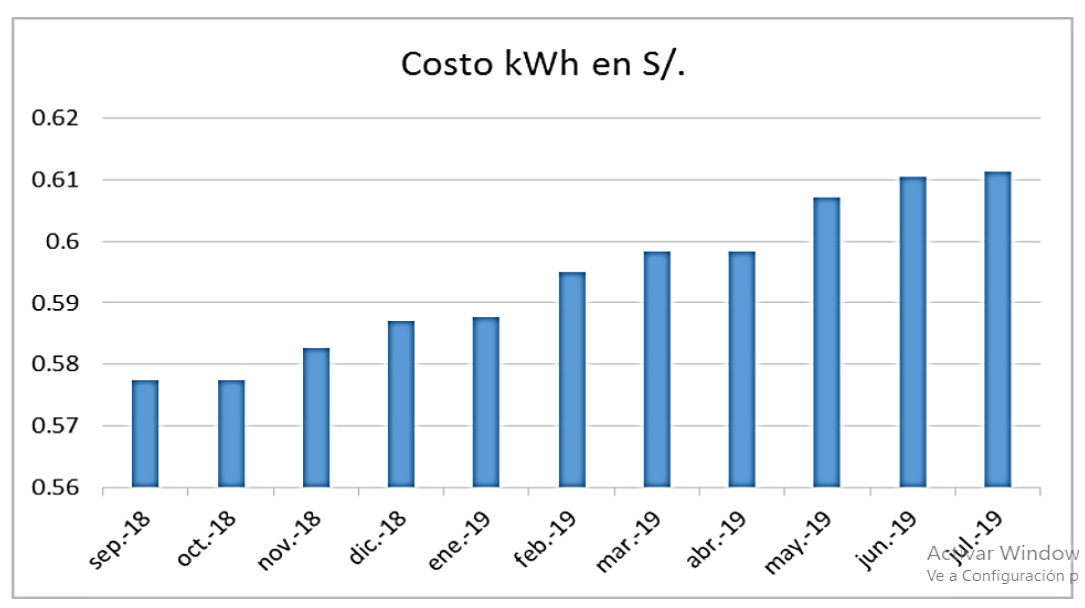

Figura 7: Tasa de crecimiento anual en el costo de energía eléctrica del sistema convencional

Fuente: López, 2019.

En esta sección del trabajo de investigación, se realizó un análisis económico con la finalidad de poder evaluar si es viable o no realizar esta propuesta en la UNTELS. Los dos parámetros que se utilizaron en este análisis económico fueron el Valor Actual Neto VAN, el cual dará el flujo de caja de los ingresos menos los egresos, y la Tasa Interna de Retorno TIR que es un porcentaje que mide la viabilidad de un proyecto; siempre que la TIR sea mayor que un $12 \%$ el proyecto será económicamente viable. Se cotizaron los precios con diferentes proveedores confiables y responsables como la empresa Fuente Alternativa Servicios e Ingeniería y la empresa Auto Solar, siempre cuidando de obtener componentes confiables. En la Tabla 7 se presenta la cotización de diferentes componentes y accesorios a emplear en el sistema de generación de energía propuesto.

Tabla 7: Cotización de componentes y accesorios

\begin{tabular}{lccc}
\hline \multicolumn{1}{c}{ Componentes y accesorios } & Cantidad & Precio unitario & Precio total \\
\hline Panel Jinko Solar 335 W & 18 & $\mathrm{~S} / 561.60$ & $\mathrm{~S} / 10,108.8$ \\
Inversor Fronius Symo 6.0-3 & 1 & $\mathrm{~S} / 8,288.3$ & $\mathrm{~S} / 8,288.30$ \\
Estructura metálica & 1 & $\mathrm{~S} / 3,508.5$ & $\mathrm{~S} / 3,508.50$ \\
Accesorios de protección, & 1 & $\mathrm{~S} / 3,902.3$ & $\mathrm{~S} / 3,902.30$ \\
llaves térmicas, cableado & & & \\
Transporte e instalación & 1 & $\mathrm{~S} / 2,229.9$ & $\mathrm{~S} / 2,229.90$ \\
\hline
\end{tabular}




\begin{tabular}{ll}
\hline SUBTOTAL & $\mathrm{S} / 25,935.0$ \\
IGV $18 \%$ & $\mathrm{~S} / 4,668.30$ \\
\hline TOTAL & $\mathrm{S} / 30,603.3$ \\
\hline
\end{tabular}

Fuente: Elaboración propia

En este caso, no se consideraron mantenimientos anuales, ya que al ser una universidad tecnológica los alumnos de la carrera de ingeniería ambiental de los ciclos VI, VII y VIII en los cursos de Desarrollo Sostenible, Energías Renovables y Tecnología Ambiental pueden realizar prácticas preprofesionales para poder fortalecer sus conocimientos adquiridos y promover la investigación en el sector de energías renovables.

Cálculo del VAN y la TIR

Para calcular los valores del Valor Actual Neto VAN y la Tasa Interna de Retorno TIR se aplican las fórmulas:

$\mathrm{VAN}=\sum_{\mathrm{t}=0}^{\mathrm{n}} \frac{\mathrm{Ft}}{(1+\mathrm{r})^{\mathrm{t}}}-\mathrm{I}$

$0=\sum_{\mathrm{t}=0}^{\mathrm{n}} \frac{\mathrm{Ft}}{(1+\mathrm{TIR})^{\mathrm{t}}}-\mathrm{I}$

en las cuales Ft es el beneficio neto de flujo anual, I es la inversión inicial, $r$ es la tasa de descuento, $\mathrm{t}$ corresponde al tiempo o periodo y n es el número de años. Para el cálculo del VAN y la TIR se utilizó Microsoft Excel y el simulador Gâbilos Software para el cálculo de los indicadores.

Se consideró que el tiempo de vida útil del sistema fotovoltaico será de 25 años. Esta estimación se obtuvo de las fichas técnicas de los componentes de las marcas seleccionadas. Además, en caso de que se solicite un préstamo a una entidad bancaria la tasa promedio es de un $10 \%$. Después era necesario saber el flujo anual en soles que sale producto del ahorro en $\mathrm{kWh}$, gracias al generador fotovoltaico. Para esto se usó el costo de kWh que cada año incrementará con una tasa de crecimiento de 0.0339 soles por la generación anual del sistema fotovoltaico, tal como se muestra en la Tabla 8.

Tabla 8: Flujo anual en soles del ahorro en $\mathrm{kWh}$ 


\begin{tabular}{|c|c|c|c|c|c|}
\hline Año & $\begin{array}{l}\text { Costo del } \\
\text { kWh }\end{array}$ & $\begin{array}{c}\text { Ahorro anual } \\
\text { en kWh }\end{array}$ & Ingresos & $\begin{array}{c}\text { Egresos } \\
\text { (Inversión } \\
\text { inicial) }\end{array}$ & $\begin{array}{c}\text { Flujo de caja } \\
\text { anual } \\
\text { acumulado }\end{array}$ \\
\hline 0 & - & - & - & $\mathrm{S} / 30,603.30$ & $-\mathrm{S} / 30,603.3$ \\
\hline 1 & 0.5939 & 9734.41 & $\mathrm{~S} / 5,781.27$ & - & $\mathrm{S} / 5,781.27$ \\
\hline 2 & 0.6278 & 9734.41 & $\mathrm{~S} / 6,111.26$ & - & S/6,111.26 \\
\hline 3 & 0.6617 & 9734.41 & $\mathrm{~S} / 6,441.26$ & - & $\mathrm{S} / 6,441.26$ \\
\hline 4 & 0.6956 & 9734.41 & $\mathrm{~S} / 6,771.26$ & - & S/6,771.26 \\
\hline 5 & 0.7295 & 9734.41 & $\mathrm{~S} / 7,101.25$ & - & $\mathrm{S} / 7,101.25$ \\
\hline 6 & 0.7634 & 9734.41 & $\mathrm{~S} / 7,431.25$ & - & $\mathrm{S} / 7,431.25$ \\
\hline 7 & 0.7973 & 9734.41 & $\mathrm{~S} / 7,761.25$ & - & $\mathrm{S} / 7,761.25$ \\
\hline 8 & 0.8312 & 9734.41 & $\mathrm{~S} / 8,091.24$ & - & $\mathrm{S} / 8,091.24$ \\
\hline 9 & 0.8651 & 9734.41 & $\mathrm{~S} / 8,421.24$ & - & $\mathrm{S} / 8,421.24$ \\
\hline 10 & 0.899 & 9734.41 & $\mathrm{~S} / 8,751.23$ & - & $\mathrm{S} / 8,751.23$ \\
\hline 11 & 0.9329 & 9734.41 & $\mathrm{~S} / 9,081.23$ & - & $\mathrm{S} / 9,081.23$ \\
\hline 12 & 0.9668 & 9734.41 & $\mathrm{~S} / 9,411.23$ & - & $\mathrm{S} / 9,411.23$ \\
\hline 13 & 1.0007 & 9734.41 & $\mathrm{~S} / 9,741.22$ & - & $\mathrm{S} / 9,741.22$ \\
\hline 14 & 1.0346 & 9734.41 & $\mathrm{~S} / 10,071.22$ & - & $\mathrm{S} / 10,071.22$ \\
\hline 15 & 1.0685 & 9734.41 & $\mathrm{~S} / 10,401.22$ & - & $\mathrm{S} / 10,401.22$ \\
\hline 16 & 1.1024 & 9734.41 & $\mathrm{~S} / 10,731.21$ & - & $\mathrm{S} / 10,731.21$ \\
\hline 17 & 1.1363 & 9734.41 & $\mathrm{~S} / 11,061.21$ & - & $\mathrm{S} / 11,061.21$ \\
\hline 18 & 1.1702 & 9734.41 & $\mathrm{~S} / 11,391.21$ & - & $\mathrm{S} / 11,391.21$ \\
\hline 19 & 1.2041 & 9734.41 & $\mathrm{~S} / 11,721.20$ & - & $\mathrm{S} / 11,721.20$ \\
\hline 20 & 1.238 & 9734.41 & $\mathrm{~S} / 12,051.20$ & - & $\mathrm{S} / 12,051.20$ \\
\hline 21 & 1.2719 & 9734.41 & $\mathrm{~S} / 12,381.20$ & - & $\mathrm{S} / 12,381.20$ \\
\hline 22 & 1.3058 & 9734.41 & $\mathrm{~S} / 12,711.19$ & - & $\mathrm{S} / 12,711.19$ \\
\hline 23 & 1.3397 & 9734.41 & $\mathrm{~S} / 13,041.19$ & - & $\mathrm{S} / 13,041.19$ \\
\hline 24 & 1.3736 & 9734.41 & $\mathrm{~S} / 13,371.19$ & - & $\mathrm{S} / 13,371.19$ \\
\hline 25 & 1.4075 & 9734.41 & $\mathrm{~S} / 13,701.18$ & - & $\mathrm{S} / 13,701.18$ \\
\hline
\end{tabular}

Fuente: Elaboración propia

El proyecto de investigación el cual propone suministrar energía limpia a la universidad se considera rentable, ya que se obtuvo un VAN de S/. 44213.06 soles y una TIR de $23.26 \%$; la TIR al ser mayor que $12 \%$ indica que el proyecto propuesto será rentable. 


\section{Caso particular: proyección para implementación de paneles solares en una vivienda promedio}

Tomando como referencia una vivienda promedio, cuyo consumo promedio mensual de energía es $181.88 \mathrm{kWh}$, tal como se muestra en la Tabla 9, con un costo de $\mathrm{kWh}$ de 0.5939 soles, se tiene un gasto mensual de S/ 108.01 soles. A continuación, se detalla el consumo de cargas y se presenta una simulación al implementar paneles solares para suministrar energía limpia y reducir costos.

Tabla 9: Consumo mensual de energía en una vivienda promedio

\begin{tabular}{|c|c|c|c|c|c|}
\hline $\begin{array}{l}\text { Artefactos } \\
\text { eléctricos }\end{array}$ & $\begin{array}{l}\text { Potencia } \\
\text { (W) }\end{array}$ & Cant. & $\begin{array}{c}\text { Horas de } \\
\text { uso }\end{array}$ & $\begin{array}{c}\text { Frecuencia de } \\
\text { uso }\end{array}$ & $\begin{array}{c}\text { Consumo } \\
\text { mensual } \\
(\mathrm{kWh})\end{array}$ \\
\hline Refrigeradora & 350 & 1 & 2 & Día & 21.00 \\
\hline Olla arrocera & 1000 & 1 & 1 & Día & 30.00 \\
\hline $\begin{array}{l}\text { Horno } \\
\text { microondas }\end{array}$ & 1100 & 1 & 1 & Día & 33.00 \\
\hline Televisor 29" & 175 & 3 & 4 & Día & 21.00 \\
\hline Computadora & 600 & 1 & 2 & Día & 36.00 \\
\hline Plancha & 1000 & 1 & 3 & Semana & 12.00 \\
\hline Licuadora & 300 & 1 & 3 & Semana & 3.60 \\
\hline Batidora & 200 & 1 & 2 & Semana & 1.60 \\
\hline DVD & 20 & 3 & 8 & Semana & 0.64 \\
\hline Impresora & 400 & 1 & 2 & Semana & 3.20 \\
\hline $\begin{array}{l}\text { Equipo de } \\
\text { sonido }\end{array}$ & 80 & 1 & 2 & Semana & 0.64 \\
\hline Lavadora & 500 & 1 & 3 & Semana & 6.00 \\
\hline Aspiradora & 600 & 1 & 2 & Semana & 4.80 \\
\hline Terma eléctrica & 1500 & 1 & 1 & Semana & 6.00 \\
\hline $\begin{array}{l}\text { Focos } \\
\text { ahorradores }\end{array}$ & 20 & 8 & 4 & Día & 2.40 \\
\hline \multicolumn{5}{|c|}{ Consumo promedio mensual en $\mathrm{kWh}$} & 181.88 \\
\hline \multicolumn{5}{|c|}{ Consumo promedio diario en Wh } & 5979.62 \\
\hline
\end{tabular}




\section{Fuente: Elaboración Propia}

Para la simulación de autoconsumo se debe elegir un porcentaje para abastecer. Al ser un sistema conectado a red y no contar con un sistema de almacenamiento de energía, se propuso un suministro de un $60 \%$ de energía limpia, es decir, se abastecerá 3587 Wh de un consumo diario de $5979.62 \mathrm{Wh}$.

Se calculó el número de paneles solares aplicando la ec. 1. Así, se obtuvo que

$$
\mathrm{N}_{\text {paneles }}=\frac{3587 * 125 \%}{335 * 4.9 * 0.9}=3.04
$$

Entonces, se dimensionó un sistema con 3 paneles, con una potencia de $335 \mathrm{~W}$ cada uno.

La potencia de entrada al inversor se calculó a partir de la ec. 2, como se presenta a continuación

$$
\text { Potencia de entrada }=38 * 8.82 * 3=1005.48 \mathrm{~W}
$$

La potencia de entrada resultante al inversor es $1005.48 \mathrm{~W}$. El inversor seleccionado para una vivienda tiene una capacidad de $1000 \mathrm{~W}$. Por lo tanto, no existirá problema, ya que el inversor tiene la capacidad de aguantar un $20 \%$ de sobrecarga y se estaría sobrecargando el inversor en un $0.54 \%$. El arreglo de los paneles fotovoltaicos estaría compuesto por una fila en paralelo y 3 paneles en serie.

Adicionalmente, se calculó la producción mensual de energía renovable, que brindará el sistema fotovoltaico para una vivienda, aplicando la ec. 5. Así, se encontró que

$$
\text { Produccion } \mathrm{kWh}-\text { mes }=1005 * 4.9 * 30 * 0.9=132.96 \mathrm{kWh}
$$

Se determinó la reducción en la huella de carbono a partir de la ec. 7. Así,

$$
\mathrm{kgCO} 2 \mathrm{eq}-\mathrm{mes}=132.96 \mathrm{kWh} * 0.615 \frac{\mathrm{kgCO} 2 \mathrm{eq}}{\mathrm{kWh}}=81.77 \mathrm{kgCO} 2 \mathrm{eq}
$$


Por lo tanto, se obtiene que una familia que implemente paneles solares podrá producir en promedio $132.96 \mathrm{kWh}$ al mes y también tendrá un ahorro en la huella de carbono de $81.77 \mathrm{kgCO} 2$; los paneles fotovoltaicos solamente ocuparán un área de $5.94 \mathrm{~m}^{2}$.

\section{Análisis económico para la implementación de paneles solares en una vivienda}

En la Tabla 10 se presenta la cotización de varios componentes y accesorios requeridos para la implementación de paneles solares en una vivienda promedio.

\section{Tabla 10: Cotización de precios}

\begin{tabular}{lccc}
\hline \multicolumn{1}{c}{ Componentes y accesorios } & Cantidad & Precio unitario & Precio total \\
\hline Panel Jinko Solar 335 W & 3 & $\mathrm{~S} / 561.60$ & $\mathrm{~S} / 1,684.80$ \\
Inversor Must Solar 1000 W & 1 & $\mathrm{~S} / 1,026.10$ & $\mathrm{~S} / 1,026.10$ \\
Estructura metálica & 1 & $\mathrm{~S} / 584.70$ & $\mathrm{~S} / 584.70$ \\
Accesorios de protección, & 1 & $\mathrm{~S} / 650.40$ & $\mathrm{~S} / 650.40$ \\
llaves térmicas, cableado & & & \\
Transporte e instalación & 1 & $\mathrm{~S} / 371.60$ & $\mathrm{~S} / 371.60$ \\
\hline SUBTOTAL & & & $\mathrm{S} / 4,317.60$ \\
IGV 18\% & & $\mathrm{S} / 947.70$ \\
\hline TOTAL & & $\mathrm{S} / 5,265.30$ \\
\hline
\end{tabular}

Fuente: Elaboración Propia

Utilizando Microsoft Excel, para calcular el VAN y la TIR, se concluyó que se puede realizar la implementación, ya que se obtuvo que el VAN es s/ 6997.49 soles y la TIR es $22.29 \%$.

\section{Resultados y Discusión}

Para el proyecto de investigación que propone suministrar energía limpia a la universidad se obtuvo, a partir de los cálculos realizados, que se logrará abastecer un promedio mensual de 811.20 kWh y una producción anual de 9734.41 kWh de energía limpia; esto gracias a un generador fotovoltaico de $6030 \mathrm{~W}$ que estará conformado por 18 paneles, cada uno de $335 \mathrm{~W}, 9$ de estos paneles en serie y 2 filas en paralelo. 
Para la ciudad de Lima, específicamente para las coordenadas de la universidad, el software PVGIS facilitó la información para determinar que la radiación solar promedio diaria es de $4.9 \mathrm{kWh} / \mathrm{m}^{2}$. En el mes de marzo se obtendrá una radiación máxima de 6.2 $\mathrm{kWh} / \mathrm{m}^{2}$ y en el mes de junio se tendrá una radiación mínima de $3.6 \mathrm{kWh} / \mathrm{m}^{2}$. Con base en la información sobre la radiación solar, se determinó que el generador fotovoltaico puede aportar $811.20 \mathrm{kWh}$ de forma mensual; aunque en el mes de junio se obtenga la menor radiación, en ningún momento, se dejará de abastecer satisfactoriamente menos del $2 \%$ propuesto con el generador fotovoltaico.

El análisis económico realizado para determinar la viabilidad del proyecto indicó que el Valor Actual Neto VAN es de S/. 44213.06 soles con una tasa de descuento del $10 \%$ en un horizonte de 25 años. También se obtuvo una Tasa Interna de Retorno TIR de 23.26 $\%$, lo que hace viable económicamente el proyecto, ya que la TIR supera el $12 \%$.

La reducción del dióxido de carbono va a ser proporcional a la producción de energía que produzca el generador fotovoltaico propuesto, ya que a mayor producción de energía limpia menor será el consumo de la energía convencional de la red eléctrica y es ahí donde se logra la reducción en la generación de dióxido de carbono. Se tendrá una reducción promedio mensual de 498.89 kgCO2eq y al año se podrá dejar de emitir $5986.66 \mathrm{kgCO} 2 \mathrm{eq}$.

\section{Conclusiones}

Satisfactoriamente se puede dimensionar de forma correcta el sistema fotovoltaico propuesto, ya que se cuenta con un potencial solar aceptable; el rango aceptable oscila entre 4.5 a $5.5 \mathrm{kWh} / \mathrm{m}^{2} \mathrm{y}$, específicamente, para las coordenadas en la UNTELS se cuenta con un potencial solar promedio de $4.9 \mathrm{kWh} / \mathrm{m}^{2}$, con lo cual el generador fotovoltaico de 18 paneles, con un área total de $35.62 \mathrm{~m}^{2}$, tendrá una producción promedio mensual de $811.20 \mathrm{kWh}$ y una producción anual de $9734.41 \mathrm{kWh}$ de energía limpia para ser consumida en las instalaciones de la UNTELS.

A partir del análisis económico realizado para determinar la viabilidad del proyecto, se encontró que el Valor Actual Neto VAN es de S/. 44213.06 soles y la Tasa Interna de Retorno TIR de $23.26 \%$, lo que hace viable económicamente el proyecto, ya que la TIR 
supera el $12 \%$. Para la viabilidad del proyecto no se debe dejar de lado otros factores, por ejemplo:

- Reducción en la generación de gases de efecto invernadero.

- Promover la investigación de los recursos energéticos renovables.

- Marketing para la institución por el uso de energías limpias.

El área del generador fotovoltaico propuesto, para 18 paneles de $335 \mathrm{~W}$ cada uno, equivale a $35.62 \mathrm{~m}^{2}$. Con esta área se podrá abastecer el $2 \%$ de la energía consumida en la universidad. Además, se podrá garantizar una reducción mensual en la emisión de dióxido de carbono de $498.89 \boldsymbol{k g C O 2 e q}$ y al año se podrá dejar de emitir 5986.66 kgCO2eq; son casi 6 toneladas de dióxido de carbono que, gracias a la energía solar, se dejarán de emitir a la atmosfera.

Se concluye que los paneles solares garantizarán durante todo el año el suministro de energía limpia, apoyando en la reducción de gases de efecto invernadero y reflejando beneficios en la reducción de la facturación mensual; esto gracias a que se suministrará mensualmente un promedio importante de energía limpia a la universidad.

\section{Referencias Bibliográficas}

Alarcón, R. y Cevallos, A. (2019). Implementación de un sistema de iluminación a nivel fotovoltaico para el área recreacional de la carrera de Ingeniería Ambiental, ESPAM MFL. Informe de trabajo de titulación, Escuela Superior Politécnica Agropecuaria de Manabi Manuel Félix López, Carrera de Medio Ambiente, Calceta.

Arias, D. (2018). Determinación de la huella de carbono en las actividades administrativas correspondientes a la municipalidad Distrital de Carhuamayo - Provincia de Junín, para controlar la emisión de gases de efecto invernadero. Tesis para optar el título profesional de Ingeniero Ambiental, Universidad Nacional Daniel Alcides Carrión, Escuela de formación profesional de Ingeniería Ambiental, Cerro de Pasco.

Caicedo Vargas, J. S. y Torres Ortega, J. A. (2020). Evaluación técnico ambiental para la implementación de un sistema fotovoltaico. Publicaciones e investigación, Universidad de La Salle, Facultad de ingeniería, Bogotá. doi:https://doi.org/10.22490/25394088.3920. 
Caminati, A., Ipanqué, C., Miranda, Z., Ruiz, F., Rojas, M. y Seminario, B. (2013). Alumbrado público con energía renovable en la universidad de Piura. Repositorio Institucional PIRHUA, Universidad de Piura, Facultad de Ingeniería, Área Departamental de Ingeniería Industrial y de Sistemas, Piura.

Fernández, J. (2018). Energías Renovables para todos. Energías Renovables.

Fernández, L. y Cervantes, A. (2017). Proyecto de diseño e implementación de un sistema fotovoltaico de interconexión a la red eléctrica en la Universidad Tecnológica de Altamira. Tesis de grado de Maestro en Energías Renovables, Universidad Tecnológica de Altamira, Carrera de mantenimiento industrial, Altamira.

Guzmán, T., Araya, F., Castro, G. y Obando, J. (2016). Uso de la energía solar en sistemas de producción agropecuaria: producción más limpia y eficiencia energética. Tecnología en Marcha, Encuentro de investigacion y extensión 2016, Costa Rica. doi:10.18845/tm.v29i8.2984

Ministerio de Energía y Minas (25 de octubre de 2018). MEM instala panel solar en su sede central para promover uso de energías renovables y la investigación científica. Obtenido de Portal web del MEM: http://www.minem.gob.pe/_detallenoticia.php.idSector=12\&idTitular=8740.

Ministerio del Ambiente (21 de Marzo de 2018). Inauguran planta solar más grande del país como parte de la respuesta peruana al cambio climático. Obtenido de Portal web MINAM: http://www.minam.gob.pe/notas-de-prensa/inauguran-plantasolar-mas-grande-del-pais-como-parte-de-la-respuesta-peruana-al-cambioclimatico/.

Osinergmin. (2019). Energías renovables: experiencia y perspectiva en la ruta del hacia la transición energética. Lima.

Polo, C., Torres, H. y De La Gala, A. (2019). Caracterización de un sistema fotovoltaico de 3,3 KW interconectado a la red eléctrica del CERT-UNJBG, Tacna: octubre 2016 - octubre 2017. Revista científica, Universidad Nacional Jorge Basadre Grohmann, Tacna.

Ramírez, O., Fernández, M. y Camblong, J. (2019). La energía solar fotovoltaica, aplicaciones de las innovaciones tecnológicas en Argentina, la legislación relacionada y sus perspectivas futuras. Libro digital, Universidad Nacional de General Sarmiento, XII Congreso de Ingeniería Industrial COINI 2019, Ciudad Autónoma de Buenos Aires. 
Ramos, H. y Rafael, L. (2014). Diseño de un sistema fotovoltaico integrado a la red de estacionamiento de la Universidad Tecnológica de Salamanca. Tesis de maestría en energías renovables, Universidad Tecnológica de Salamanca, Chihuahua.

Sánchez, S. y Franco, J. (2016). Diseño e implementación de un sistema fotovoltaico interconectado a red con soporte de almacenamiento en la Universidad Tecnológica de Pereira. Trabajo de titulación, Universidad Tecnológica de Pereira, Facultad de Tecnologías, Pereira.

Santa, E. C. (2016). Dimensionamiento de un sistema solar fotovoltaico para abastecer cargas de baja potencia y consumo en el laboratorio de Ingeniería Ambiental de la UNALM. Tesis para optar el título profesional, Universidad Nacional Agraria la Molina, Facultad de Ciencias, Lima.

Valdiviezo, P. (2014). Diseño de un sistema fotovoltaico para el suministro de energía eléctrica a 15 computadoras portátiles en la PUCP. Tesis para optar el título de Ingeniero Mecánico, Pontificia Universidad Católica del Perú, Facultad de Ciencias e Ingeniería, Lima. 